\title{
Erratum to: The Dold-Kan correspondence and coalgebra structures
}

\author{
W. Hermann B. Sore ${ }^{1}$
}

Received: 19 April 2016 / Accepted: 4 May 2016 / Published online: 19 May 2016

(C) Tbilisi Centre for Mathematical Sciences 2016

\section{Erratum to: J. Homotopy Relat. Struct. (2016) 11:67-96 DOI 10.1007/s40062-014-0096-1}

In Theorem 5.20 of original article, we have claimed to obtain a Quillen equivalence between the category of connected differential graded coalgebras and the category of connected simplicial coalgebras.

But a mistake has been recently discovered, prejudicing the proof of the announced result. Indeed, in the proof of Proposition 5.15 of original article, the identification $R T_{d}^{\prime}=T_{s}^{\prime} \Gamma$ that appears in the bijection

$$
\operatorname{ScoAlg}_{\mathrm{c}}\left(C, R T_{d}^{\prime}(N W)\right) \cong \operatorname{ScoAlg}_{\mathrm{c}}\left(C, T_{s}^{\prime} \Gamma(N W)\right)
$$

is wrong. This identification should be considered though in the context of categories of monoids. It leads to a wrong description of the functor

$$
T_{s}^{\prime}(W)=\bigoplus_{n \geq 0} W^{\widehat{\otimes} n}=I(K) \oplus W \oplus \cdots \oplus W^{\widehat{\otimes} n} \oplus \cdots
$$

as right adjoint to the functor $I_{s}^{\prime}: \mathbf{S c o A l g} \rightarrow \mathbf{S V c t}_{\mathrm{c}}$.

Communicated by Guillermo Cortinas.

The online version of the original article can be found under doi:10.1007/s40062-014-0096-1.

$凶$ W. Hermann B. Sore

hermann.sore@gmail.com

1 Département de Mathématiques et Informatique, UFR/ST, Université Polytechnique de Bobo-Dioulasso, 01 BP 1091, Bobo-Dioulasso 01, Burkina Faso 
We should instead consider Sweedler's cofree coalgebra functor as constructed in [1, Section 6.4] and extend it degreewise for a correct description of the right adjoint functor $T_{s}^{\prime}$.

Consequently, we are not able to establish the isomorphism $H_{*}\left(N T_{s}^{\prime} \Gamma(V)\right) \cong$ $H_{*}\left(T_{d}^{\prime}(V)\right)$ in Lemma 5.18 of original article and this is a crucial point for proving Theorem 5.20 of original article.

\section{Reference}

1. Sweedler, M.: Hopf Algebras. W.A. Benjamin, New York (1969) 